\title{
Clopidogrel Use and CYP2C19 Genotypes
Patients Undergoing Vascular Intervention Procedure: A Hospital-Based Study
}

\begin{abstract}
Yi-Ju Liao', Tzu-Hung Hsiao ${ }^{2-4}$, Ching-Heng Lin $\mathbb{1}^{2,3,5,6}$, Chun-Sheng Hsu ${ }^{7-10}$, Yen-Lin Chang ${ }^{1,8}$, Yu-Wei Chen (ID) ${ }^{\prime}$,

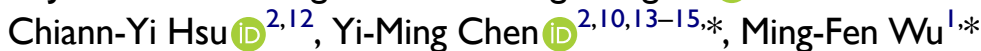

'Department of Pharmacy, Taichung Veterans General Hospital, Taichung, Taiwan; ${ }^{2}$ Department of Medical Research, Taichung Veterans General Hospital, Taichung, Taiwan; ${ }^{3}$ Department of Public Health, Fu Jen Catholic University, New Taipei City, Taiwan; ${ }^{4}$ Institute of Genomics and Bioinformatics, National Chung Hsing University, Taichung, Taiwan; ${ }^{5}$ nstitute of Public Health and Community Medicine Research Center, National Yang Ming Chiao Tung University, Taipei, Taiwan; ${ }^{6}$ Department of Industrial Engineering and Enterprise Information, Tunghai University, Taichung, Taiwan; ${ }^{7}$ Department of Physical Medicine and Rehabilitation, Taichung Veterans General Hospital, Taichung, Taiwan; ${ }^{8}$ Department of Public Health and Institute of Public Health, Chung Shan Medical University, Taichung, Taiwan; ${ }^{9}$ School of Medicine, National Defense Medical Center, Taipei, Taiwan; ${ }^{10}$ Department of Post-Baccalaureate Medicine, College of Medicine, National Chung Hsing University, Taichung, Taiwan; "'Cardiovascular Center, Taichung Veterans General Hospital, Taichung, Taiwan; ${ }^{2}$ Biostatistics Task Force of Taichung Veterans General Hospital, Taichung, Taiwan; ${ }^{13}$ Division of Allergy, Immunology and Rheumatology, Department of Internal Medicine, Taichung Veterans General Hospital, Taichung, Taiwan; ${ }^{14}$ Rong Hsing Research Center for Translational Medicine \& Ph.D. Program in Translational Medicine, National Chung Hsing University, Taichung, Taiwan; ${ }^{15}$ School of Medicine, College of Medicine, National Yang Ming Chiao Tung University, Taipei, Taiwan
\end{abstract}

*These authors contributed equally to this work

Correspondence: Yi-Ming Chen, Division of Allergy, Immunology and Rheumatology, Department of Internal Medicine, Taichung Veterans General Hospital, 1650, Section 4, Taiwan Boulevard, Xitun Dist., Taichung, 407, Taiwan, Tel +886-4-2359-2525 ext. 4400, Fax +886-4-2359-2705,

Email ymchen I@vghtc.gov.tw

Purpose: Clopidogrel is widely used in coronary artery, peripheral arterial, and cerebrovascular disease. We aimed to study the association of the CYP2C19 phenotype with cardiovascular outcomes and interventional procedures in a hospital-based population.

Patients and Methods: This cross-sectional, retrospective study enrolled patients with prior exposure to clopidogrel at the Taichung Veterans General Hospital (TCVGH) using data extracted from the Taiwan Precision Medicine Initiative (TPMI). Data on the CYP2C19 phenotype, drug-prescription profile, comorbidities, vascular intervention procedures, and hospitalization due to acute myocardial infarction (AMI) or stroke of clopidogrel users were analyzed.

Results: From the 32,728 patients in the TCVGH-TPMI cohort, we selected 2687 clopidogrel users. A total of 400 (14.9\%) clopidogrel poor metabolizers (PMs), 1235 (46.0\%) intermediate metabolizers (IMs), and 1052 (39.2\%) extensive metabolizers (EMs) were identified. The predominant loss-of-function allele is *2. In 2687 patients with clopidogrel exposure, the CYP2C19 PM phenotype was unassociated with hospitalization due to AMI or stroke after adjusting for comorbidities and carotid angiographies. Among the 1554 clopidogrel users who underwent cardiovascular intervention, $193(12.4 \%)$ received two or more types of interventional procedures. Compared with non-PMs, patients with the PM phenotype had a higher risk of multiple carotid interventions (OR: 3.13, 95\% CI: 1.19-8.22).

Conclusion: In this hospital-wide cohort, $8.2 \%$ were clopidogrel users, of which $14.9 \%$ were CYP2C19 PMs. The result of this study does not support universal genotyping of CYP2C19 in all clopidogrel users to identify risks for stroke and AMI. CYP2C19 PMs are more likely to undergo multiple carotid interventions than non-PMs. Prospective studies to investigate the association of the CYP2C19 genotype and carotid interventions and outcomes are needed to validate our results.

Keywords: pharmacogenomics, clopidogrel, CYP2C19, Taiwan precision medicine initiative, TPMI, carotid intervention

\section{Introduction}

Clopidogrel is a P2Y12 inhibitor that is widely used in the treatment of coronary artery disease (CAD), peripheral artery disease (PAD), and cerebrovascular disease. ${ }^{1-3}$ Clopidogrel is a prodrug and is metabolized into an active metabolite via 
CYP2C19. ${ }^{4}$ The US Boxed Warning on clopidogrel labels states that clopidogrel, at recommended doses, forms less active metabolites and has a reduced effect on platelet activity in CYP2C19 poor metabolizers (PMs). ${ }^{4}$ Therefore, it is recommended that medical personnel should consider another $\mathrm{P}_{2} \mathrm{Y}_{12}$ inhibitor in CYP2C19 PMs. ${ }^{4,5}$

Several studies have shown an increased risk of major adverse cardiovascular events after percutaneous coronary intervention (PCI) in CYP2C19 intermediate metabolizers (IMs) and PMs than in patients without the loss-of-function allele. $^{6}$ Medical guidelines recommend prasugrel or ticagrelor as an alternative for IMs and PMs if there is no contraindication; however, there are no universal recommendations regarding whether genetic testing should be performed. ${ }^{5}$ The TAILOR-PCI trial demonstrated the potential benefit of genotype-guided therapy in reducing major adverse cardiac events during the first 3 months after PCI. ${ }^{7}$ Real-world data has shown that the risks were most pronounced in patients with the acute coronary syndrome. ${ }^{6}$ Therefore, early intervention after PCI appears to be beneficial for acute coronary syndrome patients with the CYP2C19 phenotype.

Carriers of CYP2C19 loss-of-function alleles are at a greater risk of stroke and vascular events than noncarriers among clopidogrel users. ${ }^{8}$ Furthermore, the CYP2C19*2 loss-of-function variant allele has a significant influence on clopidogrel response in patients with carotid artery stenosis who are undergoing endarterectomy. ${ }^{9}$ CYP2C19 loss-offunction alleles (CYP2C19*2 and CYP2C19*3 alleles) were associated with a poor prognosis in patients undergoing clopidogrel therapy following a carotid artery stent placement in the Asiatic ethnic population. ${ }^{10}$ CYP2C19 genetic profiles can influence amputation-free survival and all-cause mortality in patients with PAD who are administered only clopidogrel after endovascular therapy. ${ }^{8}$ Moreover, the rate of stent patency decreased in IMs and PMs after endovascular therapy for PAD. ${ }^{6}$ However, hospital-based studies with regard to CYP2C19 phenotypes in patients with carotid intervention and PAD are limited.

This study primarily aimed to investigate the association of CYP2C19 phenotype, and cardiovascular outcomes in a hospital-based population. The secondary objective was to study whether CYP2C19 loss-of-function alleles were associated with vascular interventional procedures.

\section{Materials and Methods}

\section{Study Design}

This cross-sectional retrospective study was performed in a single medical center in Taiwan with the approval of the Ethics Committee of Taichung Veterans General Hospital (CE20316A). Each participant provided written informed consent prior to study participation. This study was conducted in accordance with the Declaration of Helsinki.

\section{Study Population}

The study population was extracted from the Taiwan Precision Medicine Initiative (TPMI), which gathered information and specimens from Taiwanese volunteers in 15 hospitals nationwide. Taichung Veterans General Hospital (TCVGH) is a tertial referral medical center that provides healthcare services to the general population in Taiwan. As the TCVGH contributed to the majority of TPMI cohort, the study population of patients aged $>20$ years who visited 28 medical and surgical outpatient clinics in TCVGH were selected for recruitment in this study from June 2019 to June 2020.

In total, 2687 patients in the TPMI cohort who were receiving clopidogrel for more than 28 days in the outpatient department of TCVGH from January 2000 to August 2020 were enrolled. We excluded patients with an undefined CYP2C19 phenotype in the TPMI and patients younger than 20 years in the TPMI.

\section{Data Collection}

We extracted data on drug-prescription profiles, comorbidities, and cardiovascular interventional procedures from the Clinical Informatics Research \& Development Center of Taichung Veterans General Hospital. Patients were classified by clopidogrel utilization into single-user, dual antiplatelet therapy (DAPT) and triple antiplatelet therapy (TAPT) which were defined as having no combination with other antiplatelet drugs for $\geq 28$ days, having a combination of clopidogrel and one antiplatelet agent for $\geq 28$ days, and having a combination of clopidogrel and two other antiplatelet agents for $\geq 28$ days, respectively. If a patient had been exposed to more than one drug in combination, all prescription records were 
retrieved, and the patient was classified as being part of the group of higher clopidogrel utilization: that is, if the patient had been exposed to a DAPT and TAPT drug combination, the patient was included in the TAPT group.

The patient's age at the first clopidogrel prescription and the department where clopidogrel was initially prescribed were recorded. Any comorbidities including heart diseases due to rheumatic heart disease, ischemic heart disease, conduction disorder and heart failure (I01, I02, I05-I09, I20-I25, I27, I30-I52/ 390-398, 410-414, 420-429), hypertension (I10-I13.2/401-404.93), diabetes mellitus (E08-E13/250), hyperlipidemia (E78.0-E78.5/414.00-414.05), chronic ischemic heart disease (I25/414.00-414.05), and stroke (I63/433.X1, 434.X1), which were diagnosed at least twice in the outpatient department or once during hospitalization were noted. The clinical outcome of hospitalization due to acute myocardial infarction (AMI) (I21-I24/410-411) and stroke (I63/433.X1, 434.X1) after first clopidogrel prescription were analyzed.

Cardiovascular interventional procedures related to the use of clopidogrel, such as cardiac catherization for CAD, cardiac artery bypass graft surgery (CABG), carotid angiography, carotid stenting, carotid endarterectomy, and extremity arteriography, were all determined by procedure codes found in the electronic health records. Among the 2687 clopidogrel users, 1554 patients with clopidogrel exposure between the first cardiovascular intervention and 28 days after the last cardiovascular intervention were then selected for the analysis dataset.

\section{CYP2C19 Genotyping and Phenotyping}

Genotyping was performed as previously described ${ }^{11}$ using the Taiwan Biobank version 2 (TWBv2) array, which is an SNP array design for the Taiwanese population. Three variants with decreased function (rs4244285(*2), rs4986893 (*3), and rs72552267 $(* 6)$ were analyzed. Patient with two loss-of-function alleles were labeled as PMs, whereas those with one loss-of-function allele were labeled as IMs. Patients without these 3 variants were labeled as extensive metabolizer (EM). Patients were then classified into PM and non-PM (both IM and EM) groups.

\section{Statistical Analysis}

Data are presented as either number (percent), mean \pm SD or medians (1st-3rd quartiles). The Kolmogorov-Smirnov test was used to confirm normal distribution of continuous variables. Comparisons were performed using the Student's $t$-test and Mann-Whitney $U$-test. Categorical data are presented as number and percentage and were compared using the chi-square or Fisher's exact test when appropriate. Multivariable logistic regression was used to investigate the association of PM phenotype with AMI and stroke in 2,87 participants on clopidogrel prescription after adjusting for patient demographics, comorbidities, and cardiovascular interventions. In addition, the association of the CYP2C19 PM phenotype with the risks of multiple carotid intervention in 1554 clopidogrel users were analyzed after adjusting for patient demographics and comorbidities. A $p$-value $\leq 0.05$ was considered statistically significant. All data were analyzed using the Statistical Package for the Social Sciences (SPSS, IBM Corp., Armonk, NY, USA) version 22.0.

\section{Results}

\section{Distribution of CYP2C19 Genotypes and Phenotypes}

From the 32,728 patients enrolled in the TPMI, 2687 clopidogrel users were selected (Figure 1). Thirty patients with an uncertain phenotype were excluded. The distribution of the predicted phenotype is shown in Table 1 . There were 400 (14.9\%) PMs, 1235 (46.0\%) IMs, and 1052 (39.2\%) EMs among the clopidogrel users. The majority of the PMs carried $* 2 / * 2(71.3 \%)$ and $* 2 / * 3(24.8 \%)$, others carried *2/*6 (1.5\%), and *3/*3 (1.3\%), whereas 5 were novel diplotypes. One participant within the 5 novel diplotypes carried genetic variations at rs4244285 (*2), rs4986893 (*3), and rs72552267 $(* 6)$. The other four patients were all homozygous for rs4244285 (*2) and heterozygous for rs72552267 (*6). As alleles $* 2, * 3$, and $* 6$ were all defined as no-function alleles, we refer to these new alleles as PMs. 


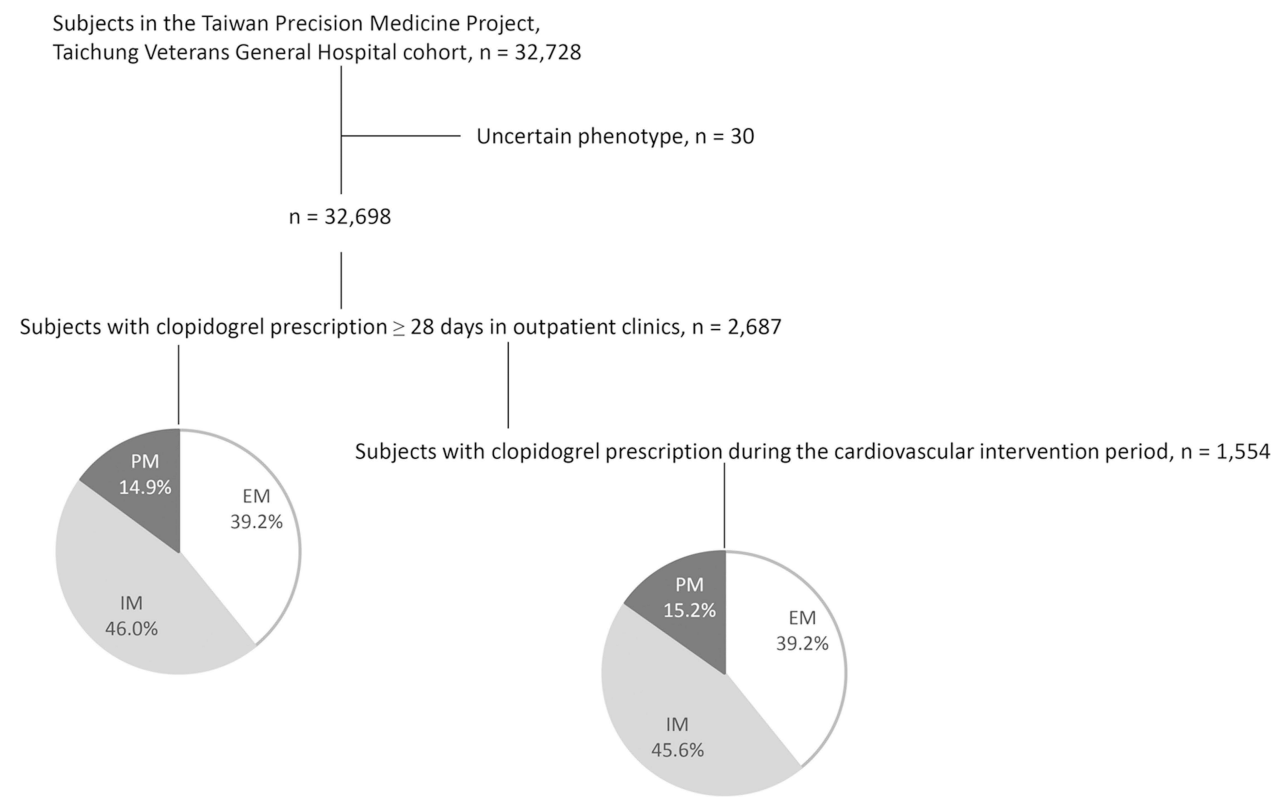

Figure I Flow diagram of the participant recruitment process in this study.

Abbreviations: EM, extensive metabolizer; IM, intermediate metabolizer; PM, poor metabolizer.

\section{Demographics of Enrolled Participants}

The patient characteristics are summarized in Table 2. The age at first prescription was $61 \pm 12$ years, and $71.16 \%$ of clopidogrel users were male. Most patients received their first prescription of clopidogrel in either the cardiovascular $(64.3 \%)$ or neurology department $(16.6 \%)$. Age, comorbidities, and location of one's first prescription department were similar among PMs and other metabolizers. In addition, 1482 (55.2\%) clopidogrel users were categorized as a part of the DAPT group. In 1656 DAPT prescription records, $87.9 \%$ were combined with aspirin, $10.1 \%$ with cilostazol, and $1.9 \%$ with dipyridamole. In the 64 TAPT prescription records, 62 were combined with aspirin and cilostazol, one with clopidogrel-cilostazol-dipyridamole, and one with aspirin-clopidogrel-dipyridamole. The distribution of each phenotype in the single-user, DAPT, and TAPT groups was similar to that in the general population. Additionally, comorbidities did not significantly differ with each phenotype.

Table I Distribution of Diplotype and Predicted Phenotype

\begin{tabular}{|l|l|l|}
\hline Phenotype & Diplotype & Clopidogrel User (\%) \\
\hline Extensive metabolizer & & $1052(39.2)$ \\
\hline & $* 1 / * 1$ & $1052(100)$ \\
\hline Intermediate metabolizer & & $1235(46.0)$ \\
\hline & $* 1 / * 2$ & $1074(87.0)$ \\
& $* 1 / * 3$ & $161(13.0)$ \\
\hline Poor metabolizer & & $400(14.9)$ \\
\hline & $* 2 / * 2$ & $285(71.3)$ \\
& $* 2 / * 3$ & $69(24.8)$ \\
& $* 2 / * 6$ & $5(1.5)$ \\
& $* 3 / * 3$ & $5(1.3)$ \\
\hline
\end{tabular}

Notes: anovel phenotype: I with rs4244285 (*2); rs4986893 (*3); rs72552267 (*6); 4 with rs4244285 (*2) homozygous and rs72552267 (*6) heterozygous. 
Table 2 Baseline Characteristics of Clopidogrel Users by CYP2C19 Phenotypes

\begin{tabular}{|c|c|c|c|c|}
\hline & \multirow[t]{2}{*}{ Total $(n=2,87)$} & \multicolumn{2}{|c|}{ CYP2CI9 Phenotype } & \multirow[t]{2}{*}{$p$-value } \\
\hline & & $P M(n=400,14.9 \%)$ & Non-PM ${ }^{b}(n=2287,85.1 \%)$ & \\
\hline $\mathrm{Age}^{\mathrm{a}}$ & $62(54-70)$ & $63(54-70)$ & $62(54-70)$ & 0.437 \\
\hline Male & 1912 (71.2\%) & $269(67.3 \%)$ & 1643 (7I.8\%) & 0.070 \\
\hline Medical department $\mathrm{t}^{\mathrm{a}}$ & & & & 0.419 \\
\hline Cardiology & $1728(64.3 \%)$ & $258(64.5 \%)$ & 1470 (64.3\%) & \\
\hline Neurology & 447 (16.6\%) & 77 (19.3\%) & $370(16.2 \%)$ & \\
\hline Vascular surgery & $136(5.1 \%)$ & 17 (4.3\%) & $119(5.2 \%)$ & \\
\hline Rheumatology & 98 (3.7\%) & $12(3.0 \%)$ & $86(3.8 \%)$ & \\
\hline Other & $278(10.4 \%)$ & $36(9.0 \%)$ & $242(10.6 \%)$ & \\
\hline Antiplatelet therapy & & & & 0.708 \\
\hline Single & II4I (42.5\%) & 177 (44.3\%) & 964 (42.2\%) & \\
\hline DAPT & I 482 (55.2\%) & $213(53.3 \%)$ & 1269 (55.5\%) & \\
\hline TAPT & $64(2.4 \%)$ & $10(2.5 \%)$ & $54(2.4 \%)$ & \\
\hline \multicolumn{5}{|l|}{ Comorbidities } \\
\hline Heart disease & $2228(82.9 \%)$ & $325(81.3 \%)$ & 1903 (83.2\%) & 0.374 \\
\hline Hypertension & 2071 (77.1\%) & 314 (78.5\%) & 1757 (76.8\%) & 0.503 \\
\hline Diabetes mellitus & 1407 (52.4\%) & $214(53.5 \%)$ & II 93 (52.2\%) & 0.661 \\
\hline Hyperlipidemia & $200 \mathrm{I}(74.5 \%)$ & 298 (74.5\%) & 1703 (74.5\%) & 1.000 \\
\hline CIHD & $|86|(69.3 \%)$ & 278 (69.5\%) & I 583 (69.2\%) & 0.957 \\
\hline Stroke & 554 (20.6\%) & 94 (23.5\%) & $460(20.1 \%)$ & 0.140 \\
\hline
\end{tabular}

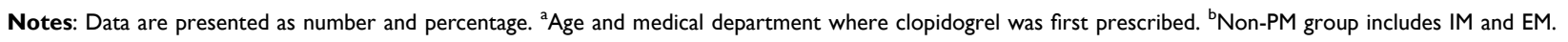
Abbreviations: DAPT, dual antiplatelet therapy; TAPT, triple antiplatelet therapy; CIHD, chronic ischemic heart disease.

\section{CYP2CI9 Phenotypes and Risks of AMI and Stroke}

Among 2687 clopidogrel users, 69 and 74 participants were hospitalized due to AMI and stroke, respectively. After adjustment for hypertension, diabetes mellitus, hyperlipidemia, and carotid intervention (Table 3), the CYP2C19 PM phenotype was found to be unassociated with hospital admission due to myocardial infarctions or cerebrovascular diseases.

\section{Clopidogrel Users with Cardiovascular Interventional Procedures}

In the study population, 1554 participants had clopidogrel exposure during the cardiovascular intervention period. The distribution of CYP2C19 phenotype in subjects with interventions was similar to that of clopidogrel users (Figure 1). Among the 1554 patients with a clopidogrel prescription during the intervention period (Table 4), 193 (12.4\%) received

Table 3 Factors Associated with Acute Myocardial Infarction and Stroke in 2687 Participants Who Were Prescribed Clopidogrel

\begin{tabular}{|c|c|c|c|c|}
\hline & \multicolumn{2}{|c|}{ Univariate } & \multicolumn{2}{|c|}{ Multivariate } \\
\hline & OR (95\% Cl) & $p$-value & OR $(95 \% \mathrm{Cl})$ & p-value \\
\hline CYP2C19 phenotype (PM vs non-PM $)$ & $1.26(0.80-1.99)$ & 0.314 & & \\
\hline Age, years & $1.00(0.98-1.01)$ & 0.667 & & \\
\hline Sex (male vs female) & $1.25(0.84-1.87)$ & 0.270 & & \\
\hline Hypertension & $3.15(1.73-5.74)$ & $<0.001$ & $2.26(1.22-4.17)$ & 0.009 \\
\hline Diabetes mellitus & $1.99(1.37-2.88)$ & $<0.001$ & $1.58(1.08-2.31)$ & 0.018 \\
\hline Hyperlipidemia & $4.02(2.16-7.49)$ & $<0.001$ & $3.36(1.79-6.32)$ & $<0.001$ \\
\hline Chronic ischemic heart disease & $1.44(0.96-2.16)$ & 0.079 & & \\
\hline Carotid angiography & $2.11(1.20-3.70)$ & 0.009 & $2.21(1.25-3.92)$ & 0.007 \\
\hline Extremity angiography & $\mathrm{I} .74(0.4 \mathrm{I}-7.49)$ & 0.455 & & \\
\hline PTCA & $1.04(0.62-1.73)$ & 0.892 & & \\
\hline
\end{tabular}

Notes: ${ }^{a}$ Non-PM group includes intermediate metabolizers and extensive metabolizers.

Abbreviations: OR, odds ratio; $\mathrm{Cl}$, confidence interval; PTCA, percutaneous transluminal coronary angioplasty; PM, poor metabolizer. 
Table 4 Cardiovascular Interventional Procedures of Clopidogrel Users by CYP2C19 Phenotypes

\begin{tabular}{|c|c|c|c|c|}
\hline & \multirow[t]{2}{*}{ Total $(n=1554)$} & \multicolumn{2}{|c|}{ CYP2C19 Phenotype } & \multirow[t]{2}{*}{ p-value } \\
\hline & & $P M(n=236)$ & Non-PM ${ }^{a}(n=13 \mid 8)$ & \\
\hline Cardiac catheterization & |47| (94.7\%) & $222(94.1 \%)$ & 1249 (94.8\%) & 0.778 \\
\hline Number of cardiac catheterization & $I(I-2)$ & $I(I-2)$ & $I(I-2)$ & 0.104 \\
\hline PTCA & $|29|$ (87.8\%) & 201 (90.5\%) & $1090(87.3 \%)$ & 0.208 \\
\hline Coronary artery bypass graft & $132(8.49 \%)$ & $14(5.9 \%)$ & II 8 (9.0\%) & 0.160 \\
\hline Number of $C A B G$ & $I(I-I)$ & $I(I-I)$ & $I(I-I)$ & 1.000 \\
\hline Carotid angiography & 148 (9.5\%) & $26(11.0 \%)$ & 122 (9.3\%) & 0.467 \\
\hline Number of carotid angiography & $I(I-I)$ & $I(I-2)$ & $I(I-I)$ & 0.010 \\
\hline Extremity angiography & $22(1.4 \%)$ & $5(2.1 \%)$ & 17 (I.3\%) & 0.363 \\
\hline Number of extremity angiography & $I(I-I)$ & $\mathrm{I}(\mathrm{I}-\mathrm{I} .5)$ & $I(I-I)$ & 0.411 \\
\hline
\end{tabular}

Notes: Data are presented as number and percentage or as median and interquartile range. ${ }^{a}$ non-PM group includes IM and EM. Abbreviations: PTCA, percutaneous transluminal coronary angioplasty; CABG, coronary artery bypass graft.

two or more types of interventional procedures; 1471 (94.66\%) underwent cardiac catherization; 132 received CABG; 148 (9.52\%) underwent carotid angiographies; and 22 (1.42\%) underwent extremity angiography.

Compared with non-PMs, CYP2C19 PMs underwent a significantly higher number of carotid angiographies (Table 4 and Figure 2). After adjustment for age and chronic ischemic heart disease, the CYP2C19 PM phenotype appeared to be a risk factor for multiple carotid interventions (OR: 3.13, 95\% CI: 1.19-8.22, Table 5).

\section{Discussion}

This hospital-based genotyping study showed that $8.2 \%$ patients were clopidogrel users, of which $14.9 \%$ were CYP2C19 PMs. Moreover, PM patients exhibited a higher risk of multiple carotid interventions than non-PMs. Our results indicate

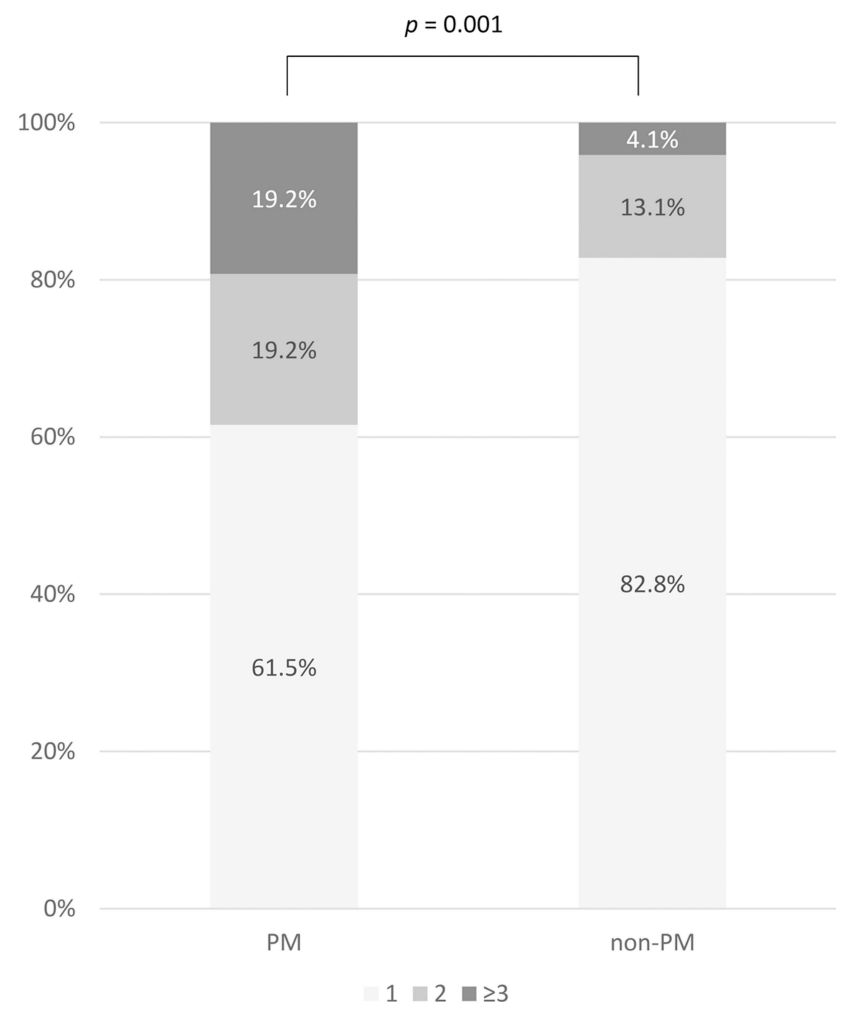

Figure 2 Comparison of the number of carotid angiographies in the CYP2C19 PM and non-PM groups. 
Table 5 Factors Associated with the Number of Carotid Angiography in Clopidogrel Users

\begin{tabular}{|l|c|c|c|c|}
\hline \multirow{2}{*}{} & \multicolumn{2}{|c|}{ Univariate } & \multicolumn{2}{c|}{ Multivariate } \\
\cline { 2 - 5 } & OR (95\% CI) & p-value & OR (95\% CI) & p-value \\
\hline Phenotype (PM vs non-PM $\left.)^{\mathrm{a}}\right)$ & $3.01(\mathrm{I} .20-7.54)$ & 0.019 & $3.13(1.19-8.22)$ & 0.020 \\
Age, years & $0.96(0.93-1.00)$ & 0.037 & $0.97(0.93-1.01)$ & \\
Sex (male vs female) & $1.08(0.44-2.67)$ & 0.863 & & \\
Hypertension & $1.27(0.44-3.67)$ & 0.656 & & \\
Diabetes mellitus & $I .45(0.65-3.26)$ & 0.365 & & \\
Hyperlipidemia & $0.62(0.27-1.42)$ & 0.261 & $0.46(0.19-1.09)$ & 0.076 \\
Chronic ischemic heart disease & $0.38(0.17-0.87)$ & 0.022 & & \\
\hline
\end{tabular}

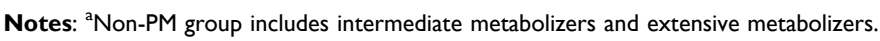

Abbreviations: $\mathrm{OR}$, odds ratio; $\mathrm{Cl}$, confidence interval; $\mathrm{PM}$, poor metabolizer.

that CYP2C19 genotyping may contribute to precision prescription in patients with high cardio-cerebrovascular risks. This finding provides insights into the potential utilization of pharmacogenomics in personalized healthcare.

According to data from the Clinical Pharmacogenetics Implementation Consortium, there are $1.5 \% \mathrm{PMs}$ in America, $2.4 \%$ in Europe, and 13\% in East Asia. ${ }^{5}$ A study of 868 patients with ischemic stroke and 557 controls demonstrated that CYP2C19 PMs constituted approximately $14 \%$ of the Taiwanese population, with more than $50 \%$ of the cohort being CYP2C19*2 carriers. ${ }^{12}$ In addition, 36.5\% EMs, 51.6\% IMs, and $11.8 \%$ PMs were identified in another study of a general population of Taiwanese ethnicity. ${ }^{13}$ Our study included $14.9 \%$ PMs and $46 \%$ IMs. Collectively, the Taiwanese, similar to other East Asians, carry a higher prevalence of CYP2C19 PM than the Caucasian population. In keeping with an earlier report, ${ }^{5}$ our results indicate that the predominant loss-of-function allele is $* 2$. However, the previous studies mainly involved participants receiving clopidogrel due to various indications. No study had focused on a hospital-based population in order to investigate the prevalence of clopidogrel users and the distribution of CYP2C19 phenotypes and genotypes. Therefore, this study is the first to show that clopidogrel has been prescribed in $8.2 \%$ patients from a tertiary referral hospital, wherein $14.9 \%$ of clopidogrel users carried PMs. This result provides a different perspective when estimating how CYP2C19 pharmacogenomic tests may impact clinical practice in hospitals.

In our study cohort of 2687 clopidogrel users, after adjusting for comorbidities and cardiovascular interventions, the CYP2C19 phenotype was unassociated with hospitalization due to stroke and AMI. In keeping with the result of a previous study, ${ }^{14}$ our finding did not support the need for universal CYP2C19 genotyping for all clopidogrel users. Kim at al. demonstrated that the CYP2C19 phenotype was only associated with the clinical outcome of clopidogrel therapy in patients with acute coronary syndrome but not in those with stable angina. ${ }^{15}$ Further study is needed to identify the patient population and types of interventional procedure that might benefit from CYP2C19 genotyping.

Our results show that $5.5 \%$ of clopidogrel users underwent carotid angiography. Compared with non-PMs, CYP2C19 PMs had a significantly higher chance of receiving additional carotid intervention. Neuroendovascular procedures for treating cerebrovascular disease are becoming the standard of care. Intraluminal stents and aneurysm coils usually need concurrent antiplatelet therapy to help reduce thromboembolic complications. For patients undergoing endovascular carotid stent placement, it is recommended that aspirin plus clopidogrel be prescribed for at least 30 days, both before and after carotid stent placement. ${ }^{2,16}$ An Asian population-based study of 241 patients undergoing carotid artery stent placement revealed that CYP2C19 loss-of-function alleles significantly affected the prognosis of patients who were treated with clopidogrel. ${ }^{10}$ Similarly, our results demonstrated that PMs were at a 3-fold higher risk of multiple carotid interventions than non-PMs. Whether tailoring antiplatelet therapy with CYP2C19 polymorphisms can reduce thromboembolic complications after a carotid artery stent procedure requires further study.

There are several limitations in this study. First, the study design is cross-sectional. Therefore, it is not possible to clarify the causal relationship between clopidogrel utilization and cardiovascular interventional procedures. Second, the TPMI project of Taichung Veterans General Hospital was initiated in 2019. Thus, patients without any outpatient visit after 2019 could not be recruited in this study. Third, unmeasured confounding factors of disease severities and patient 
demographics were not considered due to the retrospective study design. However, we demonstrated the association of multiple carotid interventions with the CYP2C19 PM phenotype after controlling for comorbidities as a confounder.

\section{Conclusion}

This hospital-wide cohort identified 8.2\% patients who were clopidogrel users, of which 14.9\% were CYP2C19 PMs. Our result does not support the need for universal genotyping of CYP2C19 in all clopidogrel users. However, CYP2C19 PMs were at higher risk of multiple carotid interventions than non-PMs. Prospective research is needed to investigate how the CYP2C19 genotype may affect the outcome of carotid interventions in patients receiving clopidogrel treatment.

\section{Acknowledgments}

We would like to thank all the participants and investigators of the Taiwan Precision Medicine Initiative. This study was funded by Academia Sinica 40-05-GMM and AS-GC-110-MD02. We would also like to thank the Biostatistics Task Force and the Clinical Informatics Research \& Development Center of Taichung Veterans General Hospital. Yi-Ming Chen and Ming-Fen Wu shared senior authorship for this study.

\section{Disclosure}

The authors report no conflicts of interest in this work.

\section{References}

1. Levine GN, Bates ER, Bittl JA, et al. 2016 ACC/AHA Guideline Focused Update on Duration of Dual Antiplatelet Therapy in Patients With Coronary Artery Disease: a Report of the American College of Cardiology/American Heart Association Task Force on Clinical Practice Guidelines. Circulation. 2016;134(10):e123-e155. doi:10.1161/CIR.0000000000000404

2. Gerhard-Herman MD, Gornik HL, Barrett C, et al. 2016 AHA/ACC guideline on the management of patients with lower extremity peripheral artery disease. J Am Coll Cardiol. 2017;69(11):e71-e126. doi:10.1016/j.jacc.2016.11.007

3. Kleindorfer DO, Towfighi A, Chaturvedi S, et al. 2021 Guideline for the Prevention of Stroke in Patients With Stroke and Transient Ischemic Attack: a Guideline From the American Heart Association/American Stroke Association. Stroke. 2021;52(7):e364-e467. doi:10.1161/ STR.0000000000000375

4. Bristol-Myers Squibb Sanofi Pharmaceuticals Partnership. Plavix ${ }^{\circledR}$ (Clopidogrel) Tablets [Prescribing Information]. Bridgewater, NJ: BristolMyers Squibb Sanofi Pharmaceuticals Partnership; 2021.

5. Scott SA, Sangkuhl K, Stein CM, et al. Clinical Pharmacogenetics Implementation Consortium guidelines for CYP2C19 genotype and clopidogrel therapy: 2013 update. Clin Pharmacol Ther. 2013;94(3):317-323. doi:10.1038/clpt.2013.105

6. Duarte JD, Cavallari LH. Pharmacogenetics to guide cardiovascular drug therapy. Nat Rev Cardiol. 2021;18(9):649-665. doi:10.1038/s41569-02100549-w

7. Pereira NL, Farkouh ME, So D, et al. Effect of Genotype-Guided Oral P2Y12 Inhibitor Selection vs Conventional Clopidogrel Therapy on Ischemic Outcomes After Percutaneous Coronary Intervention: the TAILOR-PCI Randomized Clinical Trial. JAMA. 2020;324(8):761-771. doi:10.1001/jama.2020.12443

8. Pan Y, Chen W, Xu Y, et al. Genetic polymorphisms and clopidogrel efficacy for acute ischemic stroke or transient ischemic attack: a systematic review and meta-analysis. Circulation. 2017;135(1):21-33. doi:10.1161/CIRCULATIONAHA.116.024913

9. Bačković D, Ignjatović S, Rakićević L, et al. Influence of Cyp2c19*2 Gene Variant on Therapeutic Response During Clopidogrel Treatment in Patients with Carotid Artery Stenosis. J Med Biochem. 2016;35(1):26-33. doi:10.1515/jomb-2015-0009

10. Zhu WY, Zhao T, Xiong XY, et al. Association of CYP2C19 Polymorphisms with the Clinical Efficacy of Clopidogrel Therapy in Patients Undergoing Carotid Artery Stenting in Asia. Sci Rep. 2016;6:25478. doi:10.1038/srep25478

11. Wei C-Y, Yang J-H, Yeh E-C, et al. Genetic profiles of 103,106 individuals in the Taiwan Biobank provide insights into the health and history of Han Chinese. Npj Genomic Medicine. 2021;6(1):10. doi:10.1038/s41525-021-00178-9

12. Lee YC, Liao YC, Chang FC, Huang HC, Tsai JY, Chung CP. Investigating CYP2C19 loss-of-function allele statuses and their association with stroke of different etiologies in a Taiwanese population. J Chin Med Assoc. 2019;82(6):469-472. doi:10.1097/JCMA.0000000000000101

13. Liou YH, Lin CT, Wu YJ, Wu LS. The high prevalence of the poor and ultrarapid metabolite alleles of CYP2D6, CYP2C9, CYP2C19, CYP3A4, and CYP3A5 in Taiwanese population. J Hum Genet. 2006;51(10):857. doi:10.1007/s10038-006-0034-0

14. Charfi R, Mzoughi K, Boughalleb M, et al. Response to clopidogrel and of the cytochrome CYP2C19 gene polymorphism. Tunis Med. 2018;96 (3):209-218.

15. Kim HS, Chang K, Koh YS, et al. CYP2C19 poor metabolizer is associated with clinical outcome of clopidogrel therapy in acute myocardial infarction but not stable angina. Circ Cardiovasc Genet. 2013;6(5):514-521. doi:10.1161/CIRCGENETICS.113.000109

16. Brott TG, Halperin JL, Abbara S, et al. 2011 ASA/ACCF/AHA/AANN/AANS/ACR/ASNR/CNS/SAIP/SCAI/SIR/SNIS/SVM/SVS guideline on the management of patients with extracranial carotid and vertebral artery disease. Stroke. 2011;42(8):e464-e540. doi:10.1161/ STR.0b013e3182112cc2 


\section{Publish your work in this journal}

Pharmacogenomics and Personalized Medicine is an international, peer-reviewed, open access journal characterizing the influence of genotype on pharmacology leading to the development of personalized treatment programs and individualized drug selection for improved safety, efficacy and sustainability. This journal is indexed on the American Chemical Society's Chemical Abstracts Service (CAS). The manuscript management system is completely online and includes a very quick and fair peer-review system, which is all easy to use. Visit http://www. dovepress.com/testimonials.php to read real quotes from published authors.

Submit your manuscript here: https://www.dovepress.com/pharmacogenomics-and-personalized-medicine-journal 